\section{H I G H L I G H T S}

\section{ION CHANNELS}

\section{Broken corkscrew}

The recent publication of the crystal structure of a voltage-gated $\mathrm{K}^{+}$channel is an important milestone in the study of ion channel function on at least two counts. First, it brings years of discussion on the actual structure of $\mathrm{K}^{+}$channels to an end. Second, it shows that our previous structural conception of the gating process, which was developed largely on the basis of structurefunction studies, is fundamentally wrong.

Jiang et al. solved the crystal structure of $\mathrm{KvAP}$, an archaebacterial channel that is closely related to eukaryotic $\mathrm{K}^{+}$channels. Not unexpectedly, they found that the pore region (the P loop and the S5 and S6 transmembrane domains) of KvAP was quite similar to that of KcsA, another bacterial channel for which we have structural information. The unexpected finding came from the analysis of the rest of the protein.

Until now, the accepted structural model of voltage-gated $\mathrm{K}^{+}$channels stated that S1-S4 were transmembrane helices and, owing to the presence of a series of charged residues, $\mathrm{S} 4$ was identified as the voltage sensor. In response to voltage changes, $\mathrm{S} 4$ was thought to rotate like a corkscrew inside the membrane, protected from the hydrophobic lipid environment by S1-S3, and this rotation was thought to be linked somehow to channel opening. As it turns out,
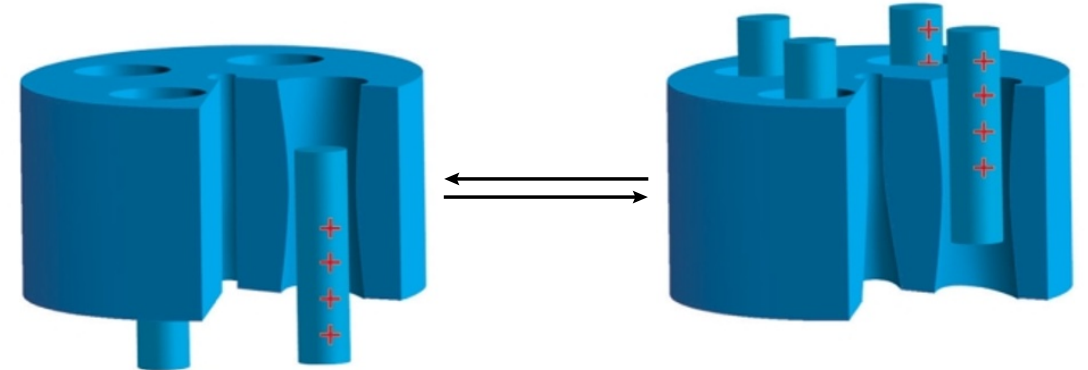

b

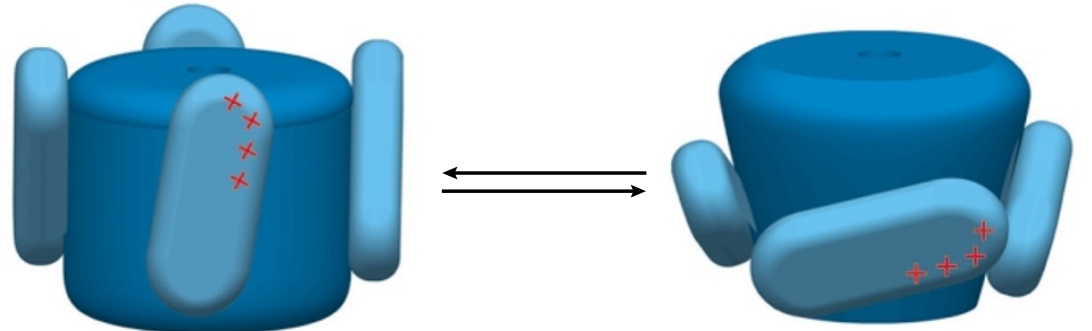

c
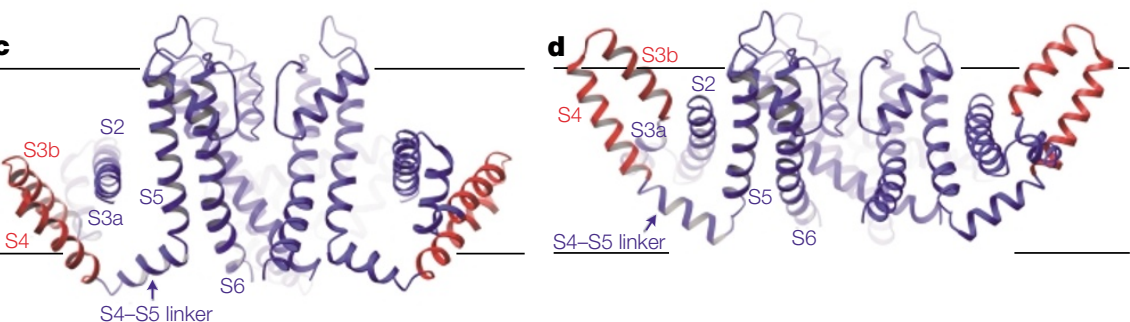

$\mathrm{K}^{+}$-channel gating. a Corkscrew model. b $\mid$Paddle model. c $\mid$Ribbon diagram of the KvAP structure in the closed state. The voltagesensor paddle is shown in red. $\mathbf{d} \mid$ KVAP in the open state, showing the large displacement of the paddle. Reproduced, with permission, from Jiang et al. Nature (c) (2003) Macmillan Magazines Ltd.

the X-ray data showed that $\mathrm{S} 4$ is perpendicular to the ion-conduction pathway, close to the intracellular face of the channel, and tightly packed against the carboxy-terminal part of S3, forming what the authors refer to as the voltage- sensor paddle. This paddle is linked to the rest of the channel through flexible loops, indicating that it might move through the membrane. This observation raises the possibility that this motion is coupled to the movements of $\mathrm{S} 5$ and

\title{
Reading the currents
}

Functional brain imaging - most commonly, positron emission tomography (PET) or functional magnetic resonance imaging (fMRI) - has become an important tool for many neuroscientists. But, despite recent

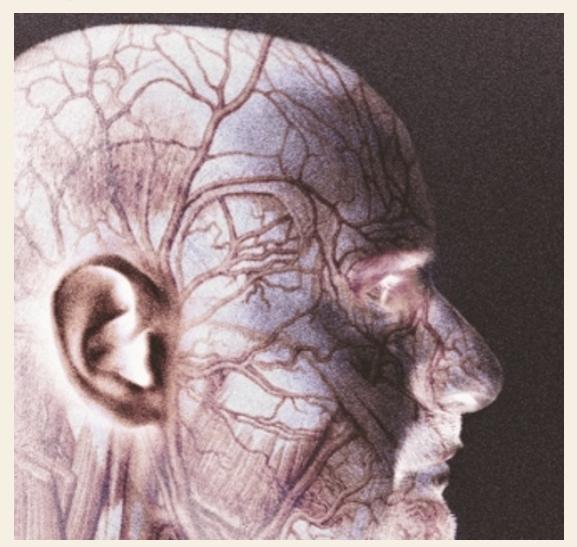

advances, it is still far from clear exactly how the changes in cerebral blood flow and oxygen metabolism that these techniques measure are related to neuronal activity. Caesar $e t$ al. have developed a new model in which to investigate this relationship, and they find that it depends largely on context.

The authors used laser Doppler flowmetry to measure local cerebral blood flow while they stimulated one or both of two inputs to cerebellar Purkinje cells. One input, carried by the climbing fibres, is excitatory, whereas the other, carried by the parallel fibres, is inhibitory. When either of these inputs was stimulated, the authors measured a frequencydependent increase in cerebral blood flow that seemed to be coupled with an increase in the postsynaptic element of the local field potential. This is consistent with previous work by Logothetis and colleagues, in which simultaneous fMRI and neurophysiological recordings showed that the fMRI signal correlated more closely with the local field potential than with spiking activity.

On the basis of a theoretical model, Caesar et al. hypothesized that imposing an inhibitory input onto an existing excitatory input would reduce the ongoing increase in cerebral blood flow, compared with that produced by the excitatory signal alone. However, when they carried out this experiment - stimulating the climbing fibres and then the parallel fibres - they found that the inhibitory input caused the cerebral blood flow to increase further. The same thing happened when they reversed the order of stimulation so that an excitatory input was added to an existing inhibitory one. However, in both cases, the combined stimulation produced an increase in cerebral blood flow that was less than the sum of the increase produced by the two inputs when each was stimulated alone.

So although the results did not agree with the authors' hypothesis, they did show that the change in cerebral blood flow caused by 
the rest of the ion-conduction pathway, leading to channel opening.

In a companion article, Jiang et al. explored the validity of this radically different gating model by replacing different residues of the paddle with cysteine, biotinylating this amino acid, and testing whether avidin, a molecule with high affinity for biotin, would bind the paddle extra- or intracellularly during channel opening. They found that avidin could bind to the $\mathrm{S} 3$ residues only from the outside of the cell and only if the channel was open. In the case of S4, avidin had access to some residues only from the outside (upon channel opening), to others only from the inside (when the channel was closed) and to a third class from both sides. These data imply that the paddle indeed experiences a large displacement across the lipid bilayer following voltage changes. This movement might simply pull the S5 domain to open the channel.

$\mathrm{So}, \mathrm{K}^{+}$-channel gating involves a fundamentally different operational principle that could not have emerged from mutational analysis alone. The publication of these two papers constitutes an important landmark for the field and will undoubtedly be hailed as one of the breakthroughs of the year.

Juan Carlos López

(2) References and links

ORIGINAL RESEARCH PAPERS Jiang, $Y$, et al. X-ray structure of a voltage-dependent $\mathrm{K}^{+}$channel. Nature 423, 33-41 (2003) | Jiang, Y. et al. The principle of gating charge movement in a voltage-dependent $\mathrm{K}^{+}$channel. Nature 423, 42-48 (2003)

stimulation of these inputs depends on the context of that stimulation. It is possible that this effect results from a refractory period that restricts the Purkinje cells' response to the additional input and is related to the electrical response properties of these neurons.

As Kim points out in a related article, this work helps to answer some unresolved questions in functional imaging. For example, the careful analysis demonstrates that it is possible to infer changes in postsynaptic field potential from haemodynamic signals, even though the latter are much slower than the former. Step by step, we are coming closer to a full understanding of just what functional imaging can tell us about brain activity. Rachel Jones

\section{(2) References and links}

ORIGINAL RESEARCH PAPER Caesar, K. et al. Context sensitivity of activity-dependent increases in cerebral blood flow. Proc. Natl Acad. Sci. USA 100, 4239-4244 (2003) FURTHER READING Heeger, D. J. \& Ress, D. What does fMRI tell us about neuronal activity? Nature Rev. Neurosci. 3 142-151 (2002) | Logothetis, N. K. et al. Neurophysiological investigation of the basis of the fMRI signal. Nature $\mathbf{4 1 2}$ 150-157 (2001) | Kim, S.-G. Progress in understanding functional imaging signals. Proc. Natl Acad. Sci. USA 100, 3550-3552 (2003)

\section{No Nogo — grow/no grow?}

Nogo is a myelin-associated protein that is expressed in the central, but not the peripheral, nervous system, and is thought to be partly responsible for the inability of central axons to regrow after injury. But three studies of Nogo-knockout mice published in Neuron, rather than clarifying the role of Nogo in preventing regeneration, have confused matters by finding different phenotypes.

Evidence from several studies has led to the view that Nogo, with other myelinassociated proteins, inhibits outgrowth of axons. Perhaps if this inhibition could be blocked or removed, injured axons in the central nervous system could regenerate. Three groups have generated mice that lack one, two or all three isoforms of Nogo, to see whether these mice show improved regeneration of central axons.

Nogo-A is the main isoform found in oligodendrocytes, so it has attracted the most attention in studies of regeneration. The first study, by Kim et al., used mice with a mutation that prevents expression of Nogo-A and B. After spinal cord injury in young adult mice, they found that the knockout mice showed increased sprouting of corticospinal axons and also improvements in motor function - a promising result.

The second study, by Simonen and colleagues, used a Nogo-A knockout mouse and found a smaller increase in axonal growth. By contrast, Zheng et al. found that neither a Nogo-A/B mutant nor a Nogo-A/B/C mutant mouse showed any improvement in axonal regeneration or sprouting after spinal cord injury.

There is no obvious explanation for the difference in results. Although Kim et al. found that sprouting was greatest in young Nogo-A/B knockout mice, rather than older adults, the mice used by Zheng and colleagues were also young. The fact that
Nogo-A knockout mice show a smaller increase in sprouting than the Nogo-A/B knockouts used by Kim and colleagues could be due to a compensatory increase in Nogo-B expression following the Nogo-A mutation, but the lack of regeneration in the mice used by Zheng et al. is puzzling. Clearly, much more work is needed before we will understand the role of Nogo in preventing regeneration; and a good starting point will be to find the reasons for the different phenotypes seen in these studies.

Rachel Jones

(2) References and links ORIGINAL RESEARCh PAPERS $\mathrm{Kim}$, J.-E. et al. Axon regeneration in young adult mice lacking Nogo-A/B. Neuron 38 187-199 (2003) | Simonen, M. et al. Systemic deletion of the myelin-associated outgrowth inhibitor Nogo-A improves regenerative and plastic responses after spinal cord injury. Neuron 38, 201-211 (2003) |Zheng, B. et al. Lack of enhanced spinal regeneration in Nogo-deficient mice. Neuron $\mathbf{3 8}$ 213-224 (2003) 